\title{
Factors associated with pediatrician attitudes over the use of complementary and traditional medicine on children in Muscat, Oman
}

\section{Muna Ahmed Al Saadoon ${ }^{1^{*}}$, Mohammed Suweilem Al Jafari ${ }^{2}$, Bader Darwish Al Dhouyani ${ }^{2}$, Syed Rizvi ${ }^{3}$}

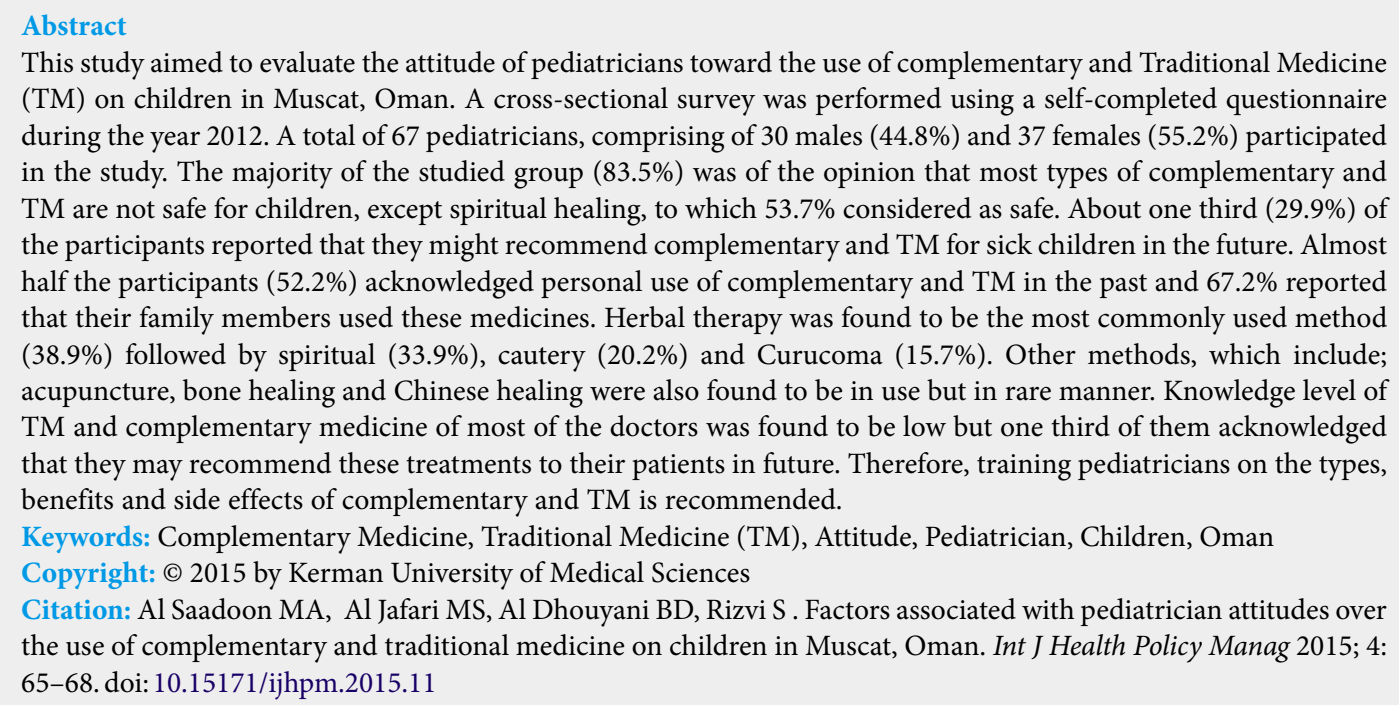
during the year 2012. A total of 67 pediatricians, comprising of 30 males (44.8\%) and 37 females (55.2\%) participated in the study. The majority of the studied group $(83.5 \%)$ was of the opinion that most types of complementary and TM are not safe for children, except spiritual healing, to which 53.7\% considered as safe. About one third (29.9\%) of the participants reported that they might recommend complementary and TM for sick children in the future. Almost half the participants (52.2\%) acknowledged personal use of complementary and TM in the past and $67.2 \%$ reported that their family members used these medicines. Herbal therapy was found to be the most commonly used method (38.9\%) followed by spiritual (33.9\%), cautery (20.2\%) and Curucoma (15.7\%). Other methods, which include; acupuncture, bone healing and Chinese healing were also found to be in use but in rare manner. Knowledge level of TM and complementary medicine of most of the doctors was found to be low but one third of them acknowledged that they may recommend these treatments to their patients in future. Therefore, training pediatricians on the types, benefits and side effects of complementary and TM is recommended.

Keywords: Complementary Medicine, Traditional Medicine (TM), Attitude, Pediatrician, Children, Oman

Copyright: (C) 2015 by Kerman University of Medical Sciences

Citation: Al Saadoon MA, Al Jafari MS, Al Dhouyani BD, Rizvi S . Factors associated with pediatrician attitudes over the use of complementary and traditional medicine on children in Muscat, Oman. Int J Health Policy Manag 2015; 4 : 65-68. doi: 10.15171/ijhpm.2015.11

Article History:

Received: 23 September 2014 Accepted: 31 December 2014 ePublished: 2 January 2015

\section{Introduction}

The term Traditional Medicine (TM) has an inclusive meaning; it includes all of the Arab-Greek medicine, ancient Chinese medicine, Indian ayurveda medicine and other forms of TM (1). Complementary or Alternative Medicine $(\mathrm{CAM})$ is the TM that is used outside the indigenous community by the other populations (2). The terms "complementary medicine" or "alternative medicine" are used inter-changeably with TM in some countries as referring to "a broad set of healthcare practices that are not part of that country's own tradition and are not integrated into the dominant healthcare system" $(1,3)$.

The use of complementary and TM has increased significantly throughout the world over the past several decades. The World Health Organization (WHO) reported that between $70 \%$ to $90 \%$ of the populations of Canada, France, Germany, and Italy have used some form of complementary and/or TM and that 110 of the 193 WHO member states reported having some type of policy in place regarding regulation and/or registration of traditional medications in 2007 (4). Studies reported half to more than two third of the medical staff have had the experience of using complementary and TM $(5,6)$.

The use of complementary and TM among children with health problems is also a recognized treatment modality worldwide (7-9). In United States, approximately 38\% of adults and $12 \%$ of children were using some forms of complementary and TM in the year 2007 (10). The selection of treatment modality is usually based on relatives and friends advice (7). There appears a positive attitude toward complementary and TM among the general population, however, it is also a fact that they have limited knowledge and have their own worries about the same. A study, conducted in Iran, reported that majority of the participants (61.7\%) expressed a desire of receiving complementary and TM services in hospitals rather than other places (11). This desire of Iranian people gets support from the model existing in Japan where TM (Kampo) was prescribed by $92.4 \%$ of physicians for cancer treatment (12). Although some studies have reported the use of complementary and TM among adult patients in Oman $(13,14)$, there are no studies that address this issue among children or medical staff. The aim of this study is to assess the attitude of pediatricians toward the use of complementary and TM on children.

\section{Methods}

A cross-sectional study was conducted in two major tertiary government hospitals in Muscat in Oman. All pediatricians (general pediatrics and subspecialist in pediatrics including neonatologists) working in the two institutes were included in the study. Pediatric residents of Oman Medical Specialty Board (OMSB) posted in these two institutes were also invited to participate. Written consent was obtained prior to completing the questionnaire. Furthermore, participants were assured that all responses would be kept confidential. The questionnaire (self-completed structured questionnaire), 
designed by the research team, was based on literature review and objectives of the research. The aim of the questionnaire was to know the attitude of pediatricians toward use of complementary and TM in treating children aged 0-12 years. The questionnaire is comprised of sections covering demographic data (age, gender, nationality, qualification, number of years of experience), participant's attitude toward use of complementary and TM on children, use of the different complementary and TM types by the participant and his or her close family members, and participant's knowledge about complementary and TM. The specific complementary and TM mentioned in the questionnaire include; traditional Chinese medicine, turmeric (yellow powder extracted from herbs and commonly used as a local Omani therapy for external application on the skin), traditional herbalism (crude plant material such as leaves, flowers, fruit, seed, stems, wood, bark, roots, rhizomes or other plant parts, which may be entire, fragmented or powdered), spiritual healing (Reading Quran phrases near a patient as a way of therapy), bone healing (a traditional method of treating fractures and dislocated joints), cautery or wasam (healing by burning the skin commonly used in Oman), other types (to be inserted by participant). All questions were closed ended with specific options provided to the respondent to select from.

Face validity of the questionnaire was assessed after receiving comments from specialists in the field and a pilot study of the questionnaire was also conducted, on 10 doctors, not participating in the study. In the light of the comments from experts and the pilot study; the questionnaire was modified to make it clearer to convey the message as intended.

After obtaining ethical approval, the questionnaire was distributed to the pediatricians of the two hospitals and the filled questionnaires were collected. This process was conducted during October 2, 2012 to April 21, 2013. Database for the study sample was created in SPSS 19.0 (IBM, Corp., Chicago, Illinois, USA). As most of the variables are categorized; percentages have been mentioned for description and Chi-Square test is used to evaluate the significance of the association between two categorized variables. $P$-value of 0.05 or less has been taken as significant.

\section{Results}

Demographic data

A total of 67 pediatricians from the two major tertiary hospitals in Muscat (response rate 69.1\%) participated in the study. The study sample includes 56.7\% Omani and 43.3\% expatriate doctors. The percentage of female pediatricians in study sample was $55.2 \%$. Omani doctors were younger and less experienced as compared to non-Omanis; with respective mean ages as 33.3 years and 41.6 years. Majority of Omani doctors (60.5\%) had clinical experience ' $<5$ years' and only $18.4 \%$ had clinical experience ' $>15$ years', the comparative percentages for non-Omani were $24.1 \%$ and $41.4 \%$ respectively.

Doctors' attitudes toward the use of complementary and Traditional Medicine (TM) on children

The majority of the doctors (83.5\%) considered complementary and TM are not safe for children and
$68.7 \%$ stated that they will never recommend it for children. However, 29.9\% were in favor of recommending it to supplement Conventional Western Medical (CWM) treatment, and $1.4 \%$ might recommend it to replace CWM. Spiritual healing was considered to be the most accepted complementary and TM modality for children (Table 1).

Factors associated with attitudes toward complementary and Traditional Medicine (TM)

Demographic factors: gender, age, number of years of experience and nationality did not display any sort of association with attitude to the use of complementary and TM.

Type of health problem: Doctor's attitude toward TM or complimentary medicine was found to be softer in cases of medical problems that have no curative therapy (44.8\%), untreatable chronic illness (43.3\%) and end stage cancer (37.3\%).

Use of complementary and TM: More than half of the pediatricians (52.2\%) reported using complementary and TM at one stage of their life. Among the pediatricians who used any type of complementary or TM; spiritual healing was the most common treatment (54.3\%), closely followed by herbs (51.4\%) then Turmeric powder (25.7\%), cauterization (11.4\%) and acupuncture (5.7\%). Almost similar pattern was observed for family members but the reported use was higher, as $70.1 \%$ doctors reported that one of their close relatives have used complementary and TM. Among the users of these medicines; most common treatment among the family members was herbs $(63.8 \%)$ followed by spiritual healing (51.1\%) then cautery (42.6\%), Turmeric powder $(21.3 \%)$ and bone healing (12.8\%). Figures 1 and 2, display the use of different complementary and TM by doctors and their family members. Use of complementary and TM by family members of pediatricians was more among Omanis (86.8\%) than non-Omanis (48.3\%) and this difference was found to be statistically significant $(P=0.002)$.

Doctors who used complementary and TM in the past were more likely (51.4\%) to recommend it for patients with end stage cancer compared with those who never used (21.9\%) and this association was found to be statistically significant $(P=0.025)$. Similar trend was observed for children with medical condition without cure and untreatable chronic illness (Figure 3). Also the doctors with close family members using complementary and TM were more likely to recommend $(42.6 \%)$ than the other group (25.0\%) but this

Table 1. Attitude of pediatricians toward the safety of complementary and TM on children

\begin{tabular}{lc}
\hline Variable & Use of method not safe for the child (\%) \\
\hline Cautery & 98.5 \\
Acupuncture & 89.6 \\
Other Chinese medicine & 100.0 \\
Traditional bone healing & 100.0 \\
Herbal medicine & 92.5 \\
Spiritual healing & 46.3 \\
\hline TM= Traditional Medicine &
\end{tabular}




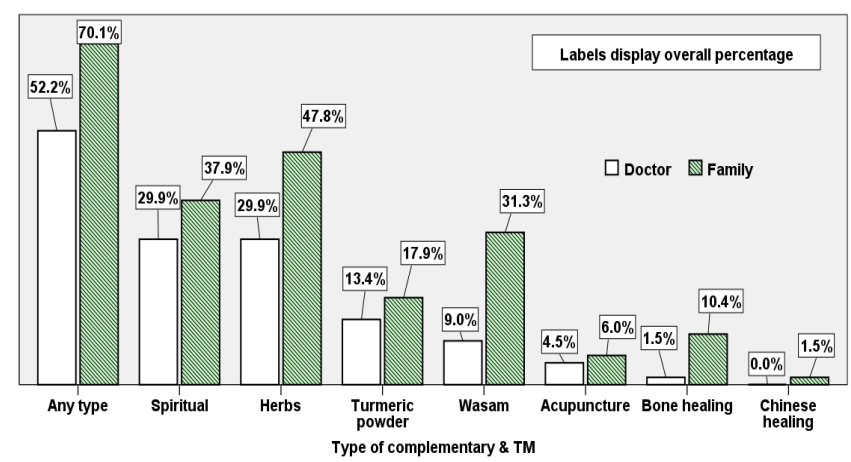

Figure 1. Use of different types of complementary and TM by the doctor and the family members

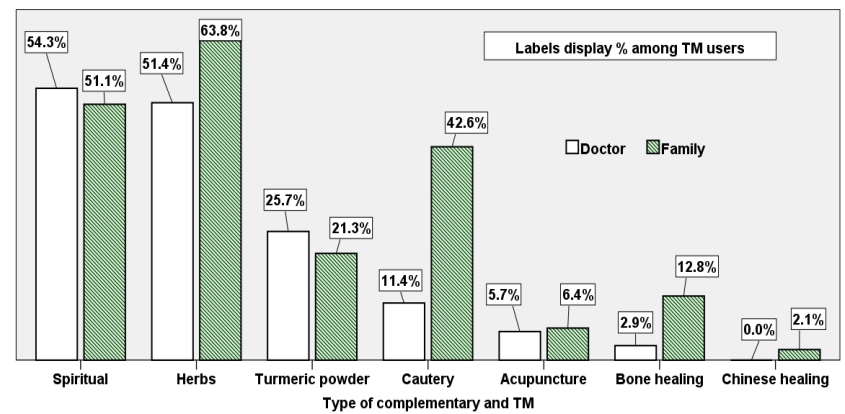

Figure 2. Use of different types of complementary and TM by the doctor and the family members (with history of using TM)

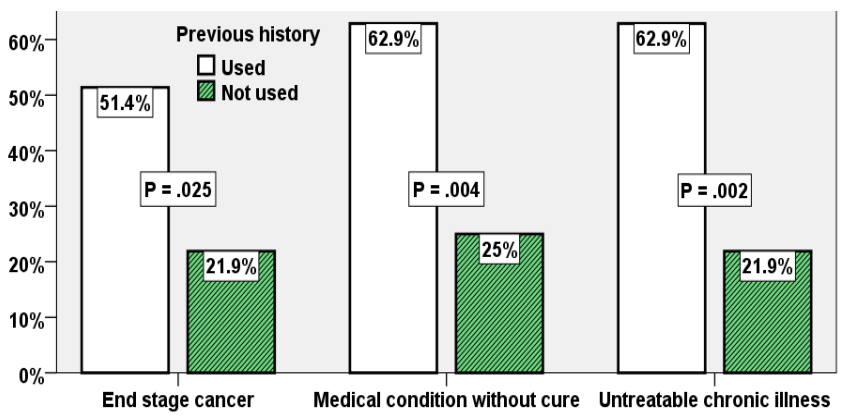

Figure 3. Positive attitude toward complementary and TM in specific health conditions in relation to previous history of use

association did not reach the desired significance $(P=0.279)$.

\section{Encountering children using complementary and Traditional} Medicine (TM)

A very high percentage (94\%) of doctors reported that during their practice in the last 5 years they encountered a child treated with complementary and TM. Most common treatment among children was cautery $(76.1 \%)$ followed by herbal medicine (59.7\%), and then spiritual treatment (53.7\%). Majority of the pediatricians (67\%) were of the opinion that use of complementary and TM adversely affected the medical condition of the child; the adverse effects include; not being compliant with prescribed medication given in the hospital (43.3\%), affecting the response to medication (16.4\%) and poisoning $(28.4 \%)$. However, few doctors $(3 \%)$ reported improvement following complementary and TM use.
On the other hand, different reasons were given by the parents to justify the use of these treatments for their children, which include; helps the child feel better (47.8\%), not satisfied with modern medicine (46.3\%), it is more natural (26.9\%) and not having side effects like modern medicine (16.5\%). Most of the time, the discussion about complimentary and TM was initiated by the doctor $(53.7 \%)$ as compared to the parent or the caregiver (46.3\%).

Pediatricians' knowledge and opinion about complementary and Traditional Medicine (TM)

Level of the knowledge of complementary and TM was measured on a self-assessed scale, ranging from $0-10$. Most of the pediatricians (86.6\%) assessed there knowledge below 5 (mean, median and mode were respectively calculated as; 3.5, 4.0 and 5.0). A large group of pediatricians (85.1\%) were of the opinion that learning and gaining knowledge of complementary medicine is necessary; most of the doctors of this group (78.9\%) desiring the knowledge to respond the queries of parents about the complementary and TM. Aim to enhance the knowledge for majority (57.9\%) of them was to convince parents to avoid these treatments. On the other hand purpose of gaining knowledge for about one third of this group (31.6\%) was to counsel the parents about the appropriate time to use these treatments.

\section{Discussion}

Attitudes toward the use of complementary and Traditional Medicine (TM) for children

This study noticed that the pediatricians with history of using these modalities were found to have a softer attitude toward the use of complementary and TM on children (Figure 3). One of the possible reasons for the same may be; they did not experience any harm in using these modalities and consequently judged it to be safe. Similar finding was reported by other studies as medical students, nurses and doctors do recommend complementary and TM to others (6).

\section{Use of complementary and Traditional Medicine (TM)}

Earlier studies reported use of these modalities among Omanis $(13,14)$, this study confirmed the previous findings. The use of complementary and TM by health professionals was reported by others and it is suggested that medical students and doctors expressed the highest use of complementary and TM compared to nurses (6).

This study did not find effect of gender or age on the frequency of use of complementary and TM, and it was equally used by Omani and non-Omani pediatricians. However, it was used more by family members of Omani doctors' as compared to families of non-Omani doctors. A possible explanation for this difference is the definition of close relative among Omani being different from the nonOmanis (among Omanis it might include wider range of relatives). The fact of being away from their country could also result in less opportunity of knowing such information about family members (thus lowering the prevalence among non-Omanis pediatricians). Another reason may be the level of social interaction and boundaries between family members which might allow more sharing of information among Omanis compared to non-Omanis. 
Encountering children using complementary and Traditional Medicine (TM)

This study found that most of the pediatricians interacted with sick children using complementary and TM, one possible reason may be the same patients reporting to the two hospitals which are located in the same city. Justifications for the use these modalities given by the parents were similar to other studies (7).

\section{Knowledge about complementary and Traditional Medicine (TM)}

This study showed that pediatrician have poor knowledge about complementary and TM which is similar to other studies findings $(6,15)$. While health service providers play an important role in helping and directing people toward healthcare and health related behaviors. It is necessary for the members of these professions to acquire reasonable knowledge about complementary and TM. The desire of acquiring the knowledge was expressed by most of the pediatrician (85.1\%), so that they could respond to queries and provide proper counseling regarding these modalities.

\section{Limitations}

The result of the study cannot be generalized as the two hospitals are located in the capital area. This study did not include the information regarding the availability of trained traditional healers within the study area. A larger sample size might have provided better comparison of other parameters.

\section{Conclusion}

More than half of the pediatricians were found to have used these modalities personally, among those who used complementary and TM, majority was in favor of recommending them in specific health conditions, viz: untreatable chronic illnesses, medical condition without cure and end stage cancer. The study found that the selfassessment of level of knowledge of these modalities was very low.

\section{Recommendations}

Considering the public interest in complementary and TM and its availability within the communities, medical organizations should consider giving more attention to this treatment modality by bridging health professional knowledge gap, empowering parents with adequate knowledge, establishing policies and regulations to control its use. The deficiency of local studies in this field also necessitates conducting more research around this topic.

\section{Ethical issues}

Ethical approval was obtained from the Ethics Committee of the College of Medicine and Health Sciences, Sultan Qaboos University (MREC 545).

\section{Competing interests}

The authors declare that they have no competing interests.

\section{Authors' contributions}

MAAS and SR participated in designing and developing the study, conducting data analysis and witting up of the manuscript. MSAJ and BDAD participated in piloting questionnaire, data collection, data entry and descriptive analysis of the data.

\section{Authors' affiliations}

${ }^{1}$ Department of Child Health, College of Medicine and Health Sciences, Sultan Qaboos University, Muscat, Oman. ${ }^{2}$ Ministry of Health, Muscat, Oman. ${ }^{3}$ Family Medicine and Public Health, College of Medicine and Health Sciences, Sultan Qaboos University, Muscat, Oman.

\section{References}

1. World Health Organization (WHO). WHO traditional medicine strategy [internet]. 2012. Available from: http://www.who.int/ medicines/publications/traditionalpolicy/en/index.html

2. World Health Organization (WHO). Traditional medicine [WWW Document]. 2012. Available from: http://www.who.int/ mediacentre/factsheets/fs134/en/

3. Gooneratne NS. Complementary and alternative medicine for sleep disturbances in older adults. Clin Geriatr Med 2008; 24: 121-8. doi: 10.1016/j.cger.2007.08.002

4. World Health Organization (WHO). Traditional medications: global situation, issues and challenges. Geneva: WHO; 2011.

5. Johnson PJ, Ward A, Knutson L, Sendelbach S. Personal use of complementary and alternative medicine (CAM) by U.S. health care workers. Health Serv Res 2012; 47: 211-27. doi: 10.1111/j.1475-6773.2011.01304.x

6. Adib-Hajbagherya M, Hoseinian M. Knowledge, attitude and practice toward complementary and traditional medicine among Kashan health care staff. Complement Ther Med 2014; 22: 12632. doi: 10.1016/j.ctim.2013.11.009

7. Gruber M, Ben-Arye E, Kerem N, Cohen-Kerem R. Use of complementary alternative medicine in pediatric otolaryngology patients: A survey. Int J Pediatr Otorhinolaryngol 2014; 78: 24852. doi: 10.1016/j.ijporl.2013.11.016

8. Birdee GS, Phillips RS, Davis RB, Gardiner P. Factors associated with pediatric use of complementary and alternative medicine. Pediatrics 2010; 125: 249-56. doi: 10.1542/peds.2009-1406

9. Farag TH, Kotloff KL, Levine MM, Onwuchekwa U, Van Eijk AM, Doh S, et al. Seeking care for pediatric diarrheal illness from traditional healers in Bamako, Mali. Am J Trop Med Hyg 2013; 89: 21-8. doi: 10.4269/ajtmh.12-0753

10. Barnes PM, Bloom B, Nahin RL. Complementary and alternative medicine use among adults and children: United States, 2007. Natl Health Stat Rep 2008; 12: 1-23. doi: 10.1037/e623942009001

11. Abolhassani H, Naseri M, Mahmoudzadeh S. A Survey of Complementary and Alternative Medicine in Iran. Chin J Integr Med 2012; 18: 409-16. doi: 10.1007/s11655-012-1124-9

12. Ito $A$, Munakata $K$, Imazu $Y$, Watanabe $K$. First nationwide attitude survey of Japanese physicians on the use of traditional Japanese medicine (Kampo) in cancer treatment. Evid Based Complement Alternat Med 2012; 2012: 957082. doi: 10.1155/2012/957082

13. Ghazanfar SA, Al-Sabahi MA. Medicinal plants of northern and central Oman. Econ Bot 1993; 47: 89-98. doi: 10.1007/ bf02862209

14. Al Saadoon M, Al-jashemi RM, Al-Farsi AM, Al-Suleimani SH, Al-Khayari HY. Medical student attitude toward traditional, complementary and alternative medicine: cross-sectional study. The Journal of Ethnobiology and Traditional Medicine 2014; 122: 900-5.

15. Mbutho NP, Gqaleni N, Korporaal CM. Traditional complementary and alternative medicine: knowledge, attitudes and practices of health care workers in HIV and AIDS clinics in Durban hospitals. Afr J Tradit Complement Altern Med 2012; 9: 64-72. doi: 10.4314/ ajtcam.v9i3s. 8 